\title{
The non-perturbative unquenched quark model
}

\author{
D.R. Entem ${ }^{1, a}$, P.G. Ortega ${ }^{2}$, and F. Fernández ${ }^{1}$ \\ ${ }^{1}$ Grupo de Física Nuclear and Instituto Universitario de Física Fundamental y Matemáticas (IUFFyM), Uni- \\ versidad de Salamanca, E-37008 Salamanca, Spain \\ ${ }^{2}$ Instituto de Física Corpuscular, Universidad de Valencia, E-46071 Valencia, Spain.
}

\begin{abstract}
In recent years states in the quarkonium spectrum not expected in the naive quark model have appeared and created a lot of interest. In the theoretical side the study of the effect of meson-meson thresholds in the spectrum have been performed in different approximations. In a quark model framework, and in the spirit of the Cornell model, when a meson-meson threshold is included, the coupling to all the quark-antiquark states have to be considered. In practice only the closest states are included perturbatively. In this contribution we will present a framework in which we couple quark-antiquark states with meson-meson states non-perturbatively, taking into account effectively the coupling to all quark-antiquark states. The method will be applied to the study of the $X(3872)$ and a comparison with the perturbative calculation will be performed.
\end{abstract}

\section{Introduction}

Since the discovery of the $X(3872)$ in 2003 [1] it became evident that the naive quark model would not be enough to describe all the hadron spectrum. This state has properties that can not be explained in such a quark model, as its decay into $J / \Psi \pi \pi$ through a $\rho$ meson, which is an isospin violating decay. However this property can be easily explained in a picture in which the state is understood as a $D D^{*}$ bound state, due to the isospin violation in the $D$ and $D^{*}$ masses and the close position of the state to the $D_{0} D_{0}^{*}$ threshold.

Already in the first studies of the charmonium spectrum it was clear that higher Fock components could play an important role. In the Cornell Model [2] the coupling between $c \bar{c}$ states and two-meson states was already included. The usual prescription is to expand the quark-antiquark wave function in eigenstates of the Hamiltonian and include perturbatively the effects of two meson loops. In this model, also the coupling between quark-antiquark states and two-meson states was performed using a microscopic model at the level of quarks. So basically, the prescription is to study the mixing between the eigenstates of the quark-antiquark Hamiltonian due to two-meson loops.

Following these ideas, and motivated by the discovery of the $X(3872)$, we started a program to study the effects of two-meson thresholds in the heavy meson spectrum. Our point of view was slightly different, since we studied the effects of the bare quark-antiquark states in the dynamics of the twomeson states, which was solved using a Schrödinger like equation. This allowed us to find new states not present in the naive quark model. Our first result was to describe the $X(3872)$ as a $D D^{*}$ molecule that was bound only when the coupling to the $2^{3} P_{1}$ bare state was considered [3].

a e-mail: entem@usal.es 
Later on, in order to study states above the lowest two-meson threshold included, we extended our dynamical equations following the framework of Baru et al. [4]. This allowed us to find the mass and width of the states as pole positions of the $T$-matrix [5]. However in all these cases we use the prescription described above for the quark-antiquark wave function.

For the $X(3872)$ it was shown that the contribution to the mass of the coupling to the $1^{3} P_{1}$ and $3^{3} P_{1}$ states is small. However some decay properties could have a sizable contribution, like the radiative decays. Including many different states makes the problem too involved and so the contribution of all the tower of bound quark-antiquark states remains an open question. For this reason we have developed a new framework in which the contribution of all the states can be obtained. To do so, the main idea is not to expand the quark-antiquark wave function in a linear combination of bare quark-antiquark states, but leave the radial wave function as an unknown of the problem solving for it.

In this contribution we will describe this framework an apply it to the study of the charmonium spectrum in the $1^{++}$channel and in particular to the $X(3872)$. As in previous studies, the coupling of two and four quark states is performed microscopically using the ${ }^{3} P_{0}$ model that has been tuned to many different strong decays [6]. We will show that when the ${ }^{3} P_{0}$ coupling is small the results are the same as the perturbative calculation.

\section{The Chiral Quark Model}

The starting point of our model is given by the interaction between quarks. The details and parameters of the model can be found in Refs. [7, 8]. Here we only sketch the main ideas of the model.

The model is based on the properties of QCD. One important aspect is that Chiral Symmetry, realized for zero quark masses, is spontaneously broken by the QCD vacuum. This has as a consequence that quarks acquire a dynamical mass, the constituent mass, that in the light quark sector is around $300 \mathrm{MeV}$. The other important outcome is that it generates pseudo-Goldstone bosons that interact with quarks. In this way pions are included in the model and gives one of the most important pieces of the interaction in the light quark sector, which generates, for example, the interaction between open charm mesons through pion exchanges. Higher pion exchanges than OPE are included effectively using scalar boson exchanges.

However we know that quarks are confined inside hadrons due to the confinement interaction. Lattice simulations have shown that a linear increasing potential is generated and at some point the string breaks due to the creation of light $q \bar{q}$ pairs. We mimic this behavior using a linear screened confinement which is linear at small distances and it saturates at a certain value when the quarkantiquark pair is separated.

However lattice simulations have also shown that there is a coulomb tail at small distances that we include through perturbative effects due to the exchange of one gluon.

An important point to notice is that these two last interactions are color interactions and so they cancel between colorless hadrons.

\section{Framework}

In this section we describe the new framework we have developed to include the contribution of all $q \bar{q}$ states of the bare Hamiltonian. We follow the same steps as in previous works $[3,5]$, being our starting points the $q \bar{q}$ interaction and the ${ }^{3} P_{0}$ model which couples the two quark and four quark sectors. The $q \bar{q}$ interactions gives us the meson states but also the interaction between mesons, that we determine using the Resonating Group Method. 
Now the physical state is written down as

$$
|\Psi\rangle=\sum_{\alpha} \mathcal{N}_{\alpha}\left|\psi_{\alpha}\right\rangle+\sum_{\beta} \chi_{\beta}(P)\left|\phi_{M 1} \phi_{M 2} \beta\right\rangle
$$

where the first term includes the quark-antiquark component and the second the two-meson component. Here $\alpha$ refers to a certain $q \bar{q}$ partial wave while $\beta$ gives all the quantum numbers of the $M_{1} M_{2}$ two meson state.

In order to make the coupling between the two sectors within the ${ }^{3} P_{0}$ model, it is convenient to solve the two body problem using the Gaussian Expansion Method basis [9]. We do that for the internal wave function of the $M_{1}$ and $M_{2}$ mesons, using then the eigenfunctions of the Hamiltonian. However for the one meson state state we use

$$
\left|\psi_{\alpha}\right\rangle=\sum_{n=1}^{n_{\max }} c_{n}^{\alpha} \phi_{n l}^{G}(r)|l j m\rangle\left|\xi_{c}\right\rangle
$$

where the coefficients $c_{n}^{\alpha}$ will be unknowns of our problem. In this way the transition amplitude given by the ${ }^{3} P_{0}$ model

$$
\left\langle\phi_{M 1} \phi_{M 2} \beta|T| \psi_{\alpha}\right\rangle=P h_{\beta \alpha}(P) \delta^{3}(P)
$$

is given by

$$
h_{\beta \alpha}(P)=\sum_{n=1}^{n_{\max }} c_{n}^{\alpha} h_{\beta \alpha}^{n}(P)
$$

The coupled equations that determines the coefficients $c_{n}^{\alpha}$ are

$$
\sum_{\alpha, n}\left[\mathcal{H}_{n^{\prime} n}^{\alpha^{\prime} \alpha}-\mathcal{G}_{n^{\prime} n}^{0 \alpha^{\prime} \alpha}(E)\right] c_{n}^{\alpha}=E N_{n^{\prime} n}^{\alpha^{\prime}} c_{n}^{\alpha^{\prime}}
$$

for the $q \bar{q}$ component and

$$
\sum_{\beta} \int H_{\beta^{\prime} \beta}\left(P^{\prime}, P\right) \chi_{\beta}(P) P^{2} d P+\sum_{\alpha} h_{\beta^{\prime} \alpha}\left(P^{\prime}\right)=E \chi_{\beta^{\prime}}\left(P^{\prime}\right)
$$

for the two meson wave function, with

$$
\mathcal{G}_{n^{\prime} n}^{0 \alpha^{\prime} \alpha}(E)=-\delta^{\alpha^{\prime} \alpha} \delta_{n^{\prime} n} \sum_{\beta} \int h_{\alpha^{\prime} \beta}^{n^{\prime}}(P) \chi_{\beta}(P) P^{2} d P
$$

Following the same procedure as in Ref. [4] we end up with the Schrödinger like equation

$$
\sum_{\alpha, n}\left[\mathcal{H}_{n^{\prime} n}^{\alpha^{\prime} \alpha}-\mathcal{G}_{n^{\prime} n}^{\alpha^{\prime} \alpha}(E)\right] c_{n}^{\alpha}=E N_{n^{\prime} n}^{\alpha^{\prime}} c_{n}^{\alpha^{\prime}}
$$

where

$$
\mathcal{G}_{n^{\prime} n}^{\alpha^{\prime} \alpha}(E)=\sum_{\beta} \int d q q^{2} \frac{\phi_{\alpha^{\prime} \beta}^{n^{\prime}}(q, E) h_{\beta \alpha}^{n}(q)}{q^{2} / 2 \mu-E-i 0^{+}}
$$

is the energy-dependent complete mass-shift matrix, define in terms of the dressed vertex

$$
\phi_{\alpha \beta^{\prime}}^{n}(E ; P)=h_{\alpha \beta^{\prime}}^{n}(P)-\sum_{\beta} \int \frac{T_{V}^{\beta^{\prime} \beta}(E ; P, q) h_{\alpha \beta}^{n}(q)}{q^{2} / 2 \mu-E} q^{2} d q
$$


where $T_{V}^{\beta \beta}\left(E ; P^{\prime}, P\right)$ is the $T$-matrix solution of the two-meson states taking into account only the residual interaction generated by the interaction between quarks that we calculated using the Resonating Group Method,

$$
T_{V}^{\beta^{\prime} \beta}\left(E ; P^{\prime}, P\right)=V^{\beta^{\prime} \beta}\left(P^{\prime}, P\right)+\sum_{\beta^{\prime \prime}} \int d P^{\prime \prime} P^{\prime \prime 2} V^{\beta^{\prime} \beta^{\prime \prime}}\left(P^{\prime}, P^{\prime \prime}\right) \frac{1}{z-E_{\beta^{\prime \prime}}\left(P^{\prime \prime}\right)} T_{V}^{\beta^{\prime \prime} \beta}\left(E ; P^{\prime \prime}, P\right)
$$

The molecular wave function is given by

$$
\chi_{\beta^{\prime}}\left(P^{\prime}\right)=-2 \mu_{\beta^{\prime}} \sum_{\alpha, n=1}^{n_{\max }} \frac{\phi_{\beta^{\prime} \alpha}\left(E ; P^{\prime}\right) c_{n}^{\alpha}}{P^{\prime 2}-k_{\beta^{\prime}}^{2}-i 0^{+}}
$$

where the normalization condition

$$
\sum_{\alpha^{\prime}, \alpha} \sum_{n^{\prime}, n=1}^{n_{\max }} c_{n^{\prime}}^{\alpha^{\prime} *} N_{n^{\prime} n}^{\alpha^{\prime} \alpha} c_{n}^{\alpha}+\sum_{\beta}<\chi_{\beta} \mid \chi_{\beta}>=1
$$

is used.

\section{Results}

In this section we present the results of the new framework applied to the $1^{++}$sector of the charmonium spectrum. Here we include $D^{0} D^{* 0}$ and $D^{ \pm} D^{* \pm}$ states (always the combination to give the correct $C$ parity is understood) with their physical masses in order to describe the isospin violation of the state.

Table 1. Mass and probabilities of the states obtained using the new framework closest to the $X(3872)$ in the $1^{++}$ charmonium sector. The first three states are obtained using the original value of the ${ }^{3} P_{0}$ strength parameter and the second finetuning this parameter to the physical mass of the $X(3872)$.

\begin{tabular}{lllllll}
\hline$\gamma^{3} P_{0}$ & $M(\mathrm{MeV})$ & $c \bar{c}$ & $D^{0} D^{* 0}$ & $D^{ \pm} D^{* \pm}$ & $\mathrm{I}=0$ & $\mathrm{I}=1$ \\
\hline \multirow{3}{*}{0.260} & 3949 & $56.71 \%$ & $22.47 \%$ & $20.82 \%$ & $43.10 \%$ & $0.25 \%$ \\
& 3867 & $30.22 \%$ & $51.37 \%$ & $18.40 \%$ & $64.72 \%$ & $5.06 \%$ \\
& 3468 & $95.70 \%$ & $2.18 \%$ & $2.12 \%$ & $4.30 \%$ & $0.0 \%$ \\
\hline \multirow{3}{*}{0.218} & 3944 & $56.82 \%$ & $22.10 \%$ & $21.07 \%$ & $42.89 \%$ & $0.51 \%$ \\
& 3871 & $3.94 \%$ & $93.46 \%$ & $2.61 \%$ & $55.79 \%$ & $38.90 \%$ \\
& 3481 & $97.10 \%$ & $1.47 \%$ & $1.43 \%$ & $2.9 \%$ & $0.0 \%$ \\
\hline
\end{tabular}

If we take the original value of the ${ }^{3} P_{0}$ strength parameter we obtain the first three states of Table 1. The highest and lowest states are the naive quark model states dressed by the two meson loops. It appears a new state that we assigned to the $X(3872)$. As some properties of the $X(3872)$ strongly depends on the $D D^{*}$ binding energy we also give the results when we fine tune the ${ }^{3} P_{0}$ strength parameter to the $X(3872)$ experimental mass.

With the $c \bar{c}$ wave function we can calculate the probabilities of the naive quark model states. This is given in Table 2 . The first state is basically a $1^{3} P_{1}$ state while the other two states are a mixture of the $2^{3} P_{1}$ state and $D D^{*}$ molecular components.

The new approach should be the same as the previous when the coupling is small. To validate the approach we show in Figure 1 the mass of the three states as a function of the ${ }^{3} P_{0}$ strength parameter $\gamma$ for the new approach (red line) compare with the previous one in which we couple $D D^{*}$ components 
Table 2. Probabilities of the naive quark model states on the physical state. The probabilities are normalized to the total $c \bar{c}$ probability.

\begin{tabular}{lllllll}
\hline$\gamma^{3} P_{0}$ & $M(\mathrm{MeV})$ & $c \bar{c}$ & $1^{3} P_{1}$ & $2^{3} P_{1}$ & $3^{3} P_{1}$ & $4^{3} P_{1}$ \\
\hline \multirow{3}{*}{0.260} & 3949 & $56.71 \%$ & $1.61 \%$ & $96.33 \%$ & $1.28 \%$ & $0.78 \%$ \\
& 3867 & $30.22 \%$ & $1.80 \%$ & $98.14 \%$ & $0.06 \%$ & $0.0 \%$ \\
& 3468 & $95.70 \%$ & $99.99 \%$ & $0.01 \%$ & $0.0 \%$ & $0.0 \%$ \\
\hline \multirow{3}{*}{0.218} & 3944 & $56.82 \%$ & $0.61 \%$ & $99.01 \%$ & $0.38 \%$ & $0.0 \%$ \\
& 3871 & $3.94 \%$ & $2.11 \%$ & $97.75 \%$ & $0.14 \%$ & $0.0 \%$ \\
& 3481 & $97.10 \%$ & $100.0 \%$ & $0.0 \%$ & $0.0 \%$ & $0.0 \%$ \\
\hline
\end{tabular}
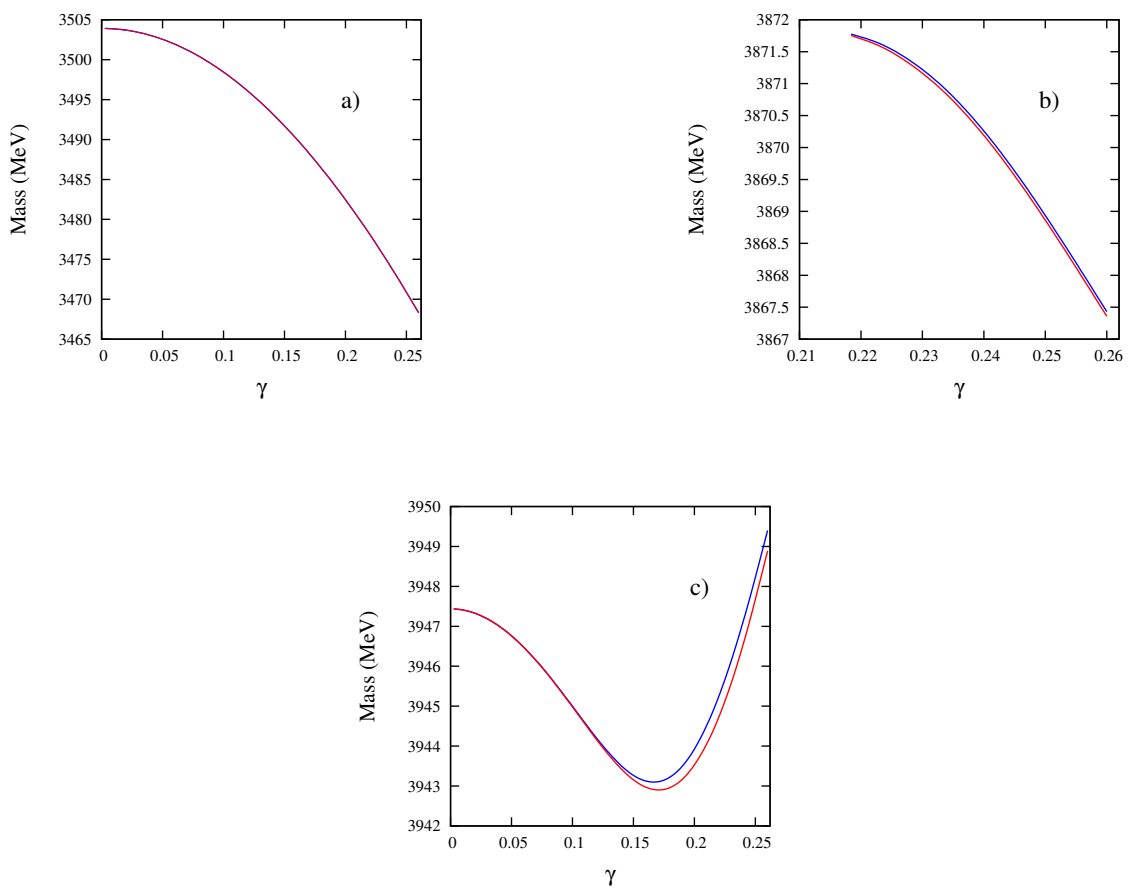

Figure 1. Mass of the three states obtained as a function of the ${ }^{3} P_{0}$ coupling parameter $\gamma$. Figure a) shows the result for the lowest lying state, b) the result for the $X(3872)$ and c) the highest. In all cases the blue line corresponds to the original approach and the red line to the new approach.

with the $1^{3} P_{1}$ and $2^{3} P_{1}$ states (blue line). Notice that for the $X(3872)$ the state disappear when the parameter decreases. The results are very similar and goes to the same value when the parameter goes to zero. The only sizable discrepancy is in the highest state due to the influence of the $3^{3} P_{1}$ state not included in the original approach. 


\section{Summary}

In this contribution we have presented a new framework in which we can study states with $q \bar{q}$ and two meson components in which no previous assumption about the $q \bar{q}$ content has to be made. The main idea is to leave the $q \bar{q}$ wave function as an unknown instead of making an expansion on the closest naive quark model states. In this way the contribution of all the bare states is taken into account effectively.

We have applied it to the study of the charmonium spectrum in the $1^{++}$channel close to the $X(3872)$. When the coupling between the one and two meson channels is small both approach are equivalent. However discrepancies may appear when the coupling is strong or when one state close to the energy under study is not included.

\section{References}

[1] S.K. Choi, S.L. Olsen, K. Abe, T. Abe, I. Adachi, B.S. Ahn, H. Aihara, K. Akai, M. Akatsu, M. Akemoto et al. (Belle Collaboration), Phys. Rev. Lett. 91, 262001 (2003)

[2] E. Eichten, K. Gottfried, T. Kinoshita, K. Lane, T.M. Yan, Phys. Rev. D17, 3090 (1978)

[3] P. Ortega, J. Segovia, D. Entem, F. Fernandez, Phys. Rev. D81, 054023 (2010)

[4] Baru, V., Hanhart, C., Kalashnikova, Yu. S., Kudryavtsev, A. E., Nefediev, A. V., Eur. Phys. J. A 44, 93 (2010)

[5] P.G. Ortega, D.R. Entem, F. Fernández, Journal of Physics G: Nuclear and Particle Physics 40, 065107 (2013)

[6] J. Segovia, D. Entem, F. Fernandez, Phys. Lett. B715, 322 (2012), 1205. 2215

[7] J. Vijande, F. Fernandez, A. Valcarce, J. Phys. G31, 481 (2005)

[8] J. Segovia, A. Yasser, D. Entem, F. Fernandez, Phys. Rev. D78, 114033 (2008)

[9] E. Hiyama, Y. Kino, M. Kamimura, Prog. Part. Nucl. Phys. 51, 223 (2003) 